\title{
Gender modifies the relationship between social networks and smoking among adults in Seoul, South Korea
}

\author{
John W. Ayers $\cdot$ C. Richard Hofstetter $\cdot$ Suzanne C. Hughes $\cdot$ \\ Hae-Ryun Park · Hee-Young Paik · Yoon Ju Song • Veronica Irvin • \\ Melbourne F. Hovell
}

Received: 3 September 2008/Revised: 19 October 2009/ Accepted: 20 January 2010/Published online: 9 March 2010

(C) The Author(s) 2010. This article is published with open access at Springerlink.com

\begin{abstract}
Objective To evaluate the interaction of gender with social network mechanisms and smoking behaviors in Seoul, South Korea, where smoking is common among men but not women.

Methods During 2002, telephone surveys were completed with 500 adults drawn from a probability sample in Seoul. Respondents described their smoking status, smoking rate (number of cigarettes smoked per day) and social networks by assessing who discouraged or encouraged smoking
\end{abstract}

\section{J. W. Ayers}

Department of Health, Behavior and Society,

Johns Hopkins Bloomberg School of Public Health,

Baltimore, USA

e-mail: jayers@jhsph.edu

J. W. Ayers - C. R. Hofstetter $(\bowtie) \cdot$ S. C. Hughes - V. Irvin · M. F. Hovell

Center for Behavioral Epidemiology and Community Health, Graduate School of Public Health, San Diego State University, 9245 Sky Park Court, Suite 230, San Diego, CA 92123, USA e-mail: rhofstet@mail.sdsu.edu

\section{R. Hofstetter}

Department of Political Science, San Diego State University, San Diego, CA, USA

\section{H.-R. Park}

Department of Food and Nutrition, Myongji University,

Kyonggi-Do, Republic of Korea

\section{H.-Y. Paik}

Department of Food and Nutrition, Seoul National University, Seoul, Republic of Korea

\section{Y. J. Song}

School of Human Ecology, The Catholic University of Korea, Bucheon-si, Republic of Korea (smoking support) or smoked (smoking models). Multivariable regressions were used for analyses.

Results Women encountered significantly less smoking support than men, 88\% (95\% confidence interval [95\% CI], 85-91) versus 70\% (95\% CI 66-73) net discouragement of smoking in their network. A difference in smoking support from 25 to $75 \%$ net discouragement was associated with a $27 \%$ (95\% CI 9-49) lower probability of smoking among women, significantly stronger $(z=3.18, p<0.01)$ than among men who had a $19 \%$ (95\% CI 8-27) lower probability of smoking. A similar difference in smoking support was associated with male smokers smoking 6.38 (95\% CI 0.86-12.30) fewer cigarettes per day, or 2,329 (95\% CI 314-4,490) fewer cigarettes per year. The later association could not be observed among women due to the small proportion of female smokers. Smoking models were not significantly associated with any smoking behaviors across genders.

Conclusions Social network mechanisms were differentially associated with the high smoking prevalence among men and low prevalence among women and should be targeted by interventions tailored to these differences.

Keywords South Koreans' health · Smoking · Social networks · Gender and health · Tobacco control

\section{Introduction}

South Korea has an historical infatuation with tobacco. Their tobacco market derives from and largely remains in government monopolies (Do and Park 2009; Corrao et al. 2000; Jee et al. 2004; Kang et al. 2003). Men often initiate smoking during mandatory military service where cigarettes have been freely distributed and smoking encouraged 
(Chang 2004; Korean Association of Smoking and Health [KASH] 2001). In addition, popular culture promotes smoking as a means of socialization (Lee 2003). The smoking climate, however, has been segregated along gender lines; smoking among men is acceptable but not among women (Chang 2004).

Estimates suggest among South Koreans that $42-65 \%$ of men, among the highest prevalence in the world (WHO 2002), and 3-6\% of women smoke (Cho et al. 2004, 2006, 2008; Gallup 2001). In 1999, about $58 \%$ of premature deaths among men and $11 \%$ among women were directly or indirectly related to smoking (Ha et al. 2003). The estimated annual cost of smoking is between $\$ 3.15$ and $\$ 4.58$ billion US dollars (Kang et al. 2003).

Previous investigations of South Koreans' smoking behaviors have largely been descriptive and therefore did not include social mechanisms that might be used to reduce smoking (Cho et al. 2004, 2006; Khang and Cho 2006; Khang et al. 2009; Chung et al. 2009; Lee 2000). These suggest higher social economic status (Cho et al. 2004, 2006; Khang et al. 2009), female gender (Khang and Cho 2006), Christian identification (Chung et al. 2009) and marital status among women (Cho et al. 2008) are associated with a lower smoking prevalence. The present study identifies behavioral mechanisms subject to interventions by examining family and friends' modeling and encouragement or discouragement of smoking.

The Behavioral Ecological Model (BEM) guided this investigation (Hovell et al. 2002, 2009; Hovell and Hughes 2009). The BEM differs from other ecological frameworks (Glass and McAtee 2006; Link and Phelan 1995; McLeroy et al. 1988), using contingency concepts from operant principles of behavior (DeGrandpre 2000; Glenn et al. 1992). Social learning has been a source of considerable historical attention (Bandura 1989; Watson 1994) and social context, partially through peer-to-peer influence, dominates the many determinants of smoking (Poland et al. 2006; Schroeder 2008).

According to the BEM, learning is a function of distal and proximal determinants. Often implicit cultural characteristics of groups, distal determinants include sanctioned behavior for genders, high and low educated groups and practices in the workplace and home. For example, smoking among female, older, educated and professional South Koreans may elicit social criticism to a greater extent than smoking among their respective counterparts (Cho et al. 2004, 2006, 2008). These may explain the differential smoking rates and suggest (hypothesis \#1) that older age, high work status and education, and female gender are associated with a lower prevalence of smoking. Proximal determinants include immediate social models that may be imitated, and positive or critical feedback from family and friends. Christakis and Fowler (2008), for example, found that smoking among network members was associated with smoking among subjects. Similarly, Hofstetter et al. found that South Korean immigrants in California were more likely to smoke (2004), try smoking (2007) and less likely to quit smoking (Ji et al. 2005) the more their friends and family provided encouragement (or less discouragement) to smoke and/or smoked themselves. Applying these associations to South Koreans suggests (hypothesis \#2) that peer-to-peer smoking support and models of smoking are associated with a higher prevalence of smoking.

The BEM and other ecological frameworks (Glass and McAtee 2006; Link and Phelan 1995; McLeroy et al. 1988) propose that distal determinants may be mediated by and/or moderate more proximal determinants. The mediation perspective suggests any proximal determinant may, in part, be a result of distal determinants. For example, gender differentials for smoking norms in South Korea suggest that (hypothesis 3a) women will be more likely than men to report a net discouragement for smoking in their network. The moderation perspective suggests the association between any proximal determinant and smoking is dependent on the distal context they are nested in. For example, in addition to discouraging smoking among women, South Korean culture places women in positions of dependence and thereby greater compliance with others (Min 2001; Jee et al. 1999), which may then increase compliance with social models and encouragement or discouragement of smoking. As a result (hypothesis 3b) peer-to-peer smoking support and models of smoking will be more influential among women than men.

\section{Methods}

Hypotheses were tested with data collected from Seoul, South Korea. Seoul is a city of approximately 9.8 million in 2000 , which is $21.4 \%$ of the national population (STATKOREA 2002). The survey instrument was developed in English and translated into Korean with the assistance of co-investigators in Seoul and California, USA. The EnglishKorean translation process was repeated to optimize isomorphism between concepts in the Korean and English languages. Focus groups led by an interview supervisor with extensive experience were used to ensure that concept meanings were accurately rendered in the South Korean translations. Fore and back translations were conducted and discussed iteratively until collaborators agreed on a final instrument, which was piloted to ensure appropriateness.

Telephone interviews were completed with 500 adults (18 years and over) residing in households that could be contacted by residential telephone in metropolitan Seoul. Interviews were administered to 248 men and 252 women. 
Random digit dialing (RDD) procedures (with stratification by gender and by each of the city's 27 telephone regions) were used. First, a list of 5,000 numbers distributed across the 27 telephone districts was produced by randomly sampling from directories proportional to the number of residential numbers in each telephone region. Second, a constant (1) was added to the suffix of each of these numbers and the resulting list then sorted in random order, producing the RDD sampling frame. Interview quotas for each calling district were established by multiplying 500 (the desired N) by the proportion of total telephone numbers in each of the 27 telephone regions. Interviews were then conducted with the household adult with "the most recent birthday" (Frey 1989). The mean interview length was 26 min [standard deviation $(\mathrm{SD})=19$ ].

Data were collected by a trained staff of graduate students at Myongi University under the supervision of the project co-investigator during late summer and early fall of 2002. Calls were made primarily during the evenings and all day on weekends. Up to five callbacks were made to each residence until interviews were completed, the targeted respondent refused the interview, or the number was found to be non-residential. A total of 8,817 calls were made, including callbacks, 175 calls were made to machines with no response, 3,617 no answer (including repeated callbacks), 841 disconnects, and 194 faxes. The cooperation rate (percent of completions of the total eligible respondents reached) was $41 \%$. This rate is comparable to other studies, where meta-analysis suggests a mean cooperation rate of $48 \%(S D=20)$ (Baruch 1999). Sample demographics approximated census demographics for Seoul. For example, the maximum deviations between sample and population (STAT-KOREA 2002) was an under-representation of $1 \%$ for females 40 and over and $3 \%$ for males $40-59$ and over-representation of $1 \%$ for younger and older males and $2 \%$ for females aged 20-39. The age by gender distribution of these data did not deviate significantly from that of the population distribution; $X_{(2)}^{2}=3.71, p<0.16$. The Institutional Review Boards of San Diego State University and Myongji University approved all procedures.

\section{Measures}

Current smoking status was measured using CDC criteria; smoked 100 cigarettes in their lifetime and currently smoke "everyday" or "some days" (USDHS 1996). Smoking rate among current smokers indicates the mean number of cigarettes smoked per day by smokers; derived from responses to: "On how many of the past 30 days did you smoke cigarettes?" and "On the days you smoke, about how many cigarettes do you usually smoke per day?" The number of days smoked was multiplied by the usual number of cigarettes smoked on smoking days with the resulting product divided by 30 .

The survey instrument included questions on egocentric social networks to identify social influences of smoking (Smith and Christakis 2008). Respondents were asked about alters (persons with whom the respondent had a relationship), and how these persons encouraged, discouraged, or modeled smoking using a pre-generated list of possible social relationships. This is among the most common forms of network analysis (Marsden 1990; Marsden 2005; Smith and Christakis 2008) and, despite some skepticism, has strong predictive validity (Marsden 1990, 2005). Following procedures used by Reifman et al. (2006), two indexes of social influence were formed (smoking support and smoking models) that represent the general density of encouragement or discouragement for, and models of, smoking in respondents' social networks.

\section{Smoking support}

A social support for smoking scale was formed by computing the degree to which respondents were discouraged or encouraged to smoke by spouse, parents, siblings, friends, children, grandparents, and aunts or uncles. The number of persons discouraging smoking was subtracted from the number of persons encouraging smoking, the difference was then divided by the number of observed ties in the respondent's social network. The index had a theoretical range of -1 to +1 , with -1 indicating $100 \%$ discouragement for smoking, +1 indicating $100 \%$ encouragement for smoking and 0 values indicate equal encouragement and discouragement.

\section{Smoking models}

Smoking models were measured by counting the number of persons (spouse, parents, siblings, children, grandparents, aunts and uncles, teachers, children's friends, and other persons) that respondents reported "... regularly smoke cigarettes" divided by the number of observed ties in respondent's social network. The resulting index had a theoretical range of 0 to +1 , with 0 indicating $0 \%$ and +1 indicating $100 \%$ of persons in the respondent's network smoked.

\section{Covariates}

Education was measured as years of formal education completed, and age as years. Occupation was computed by coding respondents into four dummy indicators: students, laborer, professional, or unemployed/retired; the latter was the reference category. 


\section{Analysis}

Estimates were computed using Stata 10.1 64-bit MP. Descriptive characteristics of the sample were appraised with comparison of means by gender using two sample $t$ tests assuming unequal variances. Predictors of smoking status, using logistic regression, and smoking rates among current smokers, using least squares regression, were evaluated. Skewness in smoking rate was constrained by computing and fitting models for the natural logarithm (Carroll and Ruppert 1988). Analysis of women's smoking rate was not feasible given very few smoked $(N=21)$. To make regression coefficients for smoking support and models interpretable, these indexes were multiplied by 10 so each unit change is a $10 \%$ increase/decrease in smoking support or models. A spline term was added to the model, to correct for nonlinearity in age, with the knot specified at 30 years so "age" indicates the average slope for age before 30 and "age+" the average slope for age at or after 30 (Marsh and Cormier 2001).

Given imbalance for predictor variables (e.g., women and men have very different distributions for variables such as smoking support), multivariable analyses were stratified by gender. Stratified analysis facilitates a simple presentation of differences in effect sizes for predictors by gender (Kraemer et al. 2006); though hypothesized moderation was formally tested by including interaction terms in a pooled analysis with adjustment for possible imbalance (Brambor et al. 2006).

Predicted quantities of interest, predicted probability of smoking or expected value on the smoking rate scale, were calculated using the estimates from the multivariable analysis by simulation using 1,000 randomly drawn sets of estimates from a sampling distribution with mean equal to the maximum likelihood point estimates and variance equal to the variance covariance matrix of the estimates, with all other predictors held at their mean values (King et al. 2000). All tests were two-tailed $p<0.05$.

\section{Results}

Respondents' mean age was 38 years (95\% Confidence interval [95\% CI], 36.91, 39.47), ranging from 18 to 82 years. Approximately $60 \%$ were married and respondents' mean years of formal education was 12.98 $(\mathrm{SD}=2.92)$ with $49 \%$ professional workers, $8 \%$ laborers, $19 \%$ students, and $23 \%$ unemployed (Table 1).

Mean smoking support was 79\% (95\% CI 77-82) net discouragement ranging from -100 to $33 \%$, suggesting the typical respondent encounters more discouragement than encouragement to smoke. Consistent with hypothesized expectations, women encountered significantly $(t=7.63$, $p<0.01)$ more discouragement for smoking than men, $88 \%$ (95\% CI; 85-91) versus 70\% (95\% CI 66-73) net discouragement. The distribution of smoking support also differed in a theoretically meaningful way between genders. No woman reported more encouragement than discouragement, while some men had more encouragement than discouragement but never more than $33 \%$ net encouragement on the scale. The average respondent reported that about $48 \%(95 \%$ CI $46-51)$ of alters in their

Table 1 Sample characteristics

\begin{tabular}{|c|c|c|c|c|c|c|c|c|c|}
\hline & \multicolumn{3}{|c|}{ Entire sample } & \multicolumn{3}{|l|}{ Men } & \multicolumn{3}{|l|}{ Women } \\
\hline & Mean & $95 \%$ CI & $N$ & Mean & $95 \% \mathrm{CI}$ & $N$ & Mean & $95 \% \mathrm{CI}$ & $N$ \\
\hline Smoker $^{\mathrm{a}}$ & 0.332 & $0.291,0.373$ & 500 & 0.605 & $0.544,0.666$ & 248 & 0.063 & $0.033,0.094$ & 252 \\
\hline Smoking rate ${ }^{\mathrm{a}}$ & 16.431 & $14.784,18.077$ & 178 & 17.276 & $15.552,19.001$ & 157 & 10.110 & $5.220,14.999$ & 21 \\
\hline Smoking support ${ }^{\mathrm{a}}$ & -0.790 & $-0.816,-0.765$ & 500 & -0.695 & $-0.734,-0.657$ & 248 & -0.884 & $-0.914,-0.854$ & 252 \\
\hline Smoking models & 0.483 & $0.457,0.510$ & 499 & 0.481 & $0.443,0.519$ & 248 & 0.486 & $0.448,0.524$ & 251 \\
\hline Women & 0.504 & $0.460,0.548$ & 500 & - & - & - & - & - & - \\
\hline Years of education & 12.990 & $12.732,13.248$ & 495 & 13.304 & $12.964,13.643$ & 247 & 12.677 & $12.290,13.065$ & 248 \\
\hline Married $^{\mathrm{a}}$ & 0.600 & $0.557,0.644$ & 498 & 0.524 & $0.462,0.587$ & 248 & 0.676 & $0.618,0.734$ & 250 \\
\hline Professional & 0.492 & $0.448,0.536$ & 500 & 0.548 & $0.486,0.611$ & 248 & 0.437 & $0.375,0.498$ & 252 \\
\hline Laborer $^{\mathrm{a}}$ & 0.080 & $0.056,0.104$ & 500 & 0.113 & $0.073,0.153$ & 248 & 0.048 & $0.021,0.074$ & 252 \\
\hline Student & 0.192 & $0.157,0.227$ & 500 & 0.238 & $0.185,0.291$ & 248 & 0.147 & $0.103,0.191$ & 252 \\
\hline Retired/not working ${ }^{a}$ & 0.236 & $0.199,0.273$ & 500 & 0.101 & $0.063,0.139$ & 248 & 0.369 & $0.309,0.429$ & 252 \\
\hline Age & 38.188 & $36.908,39.468$ & 500 & 37.637 & $35.775,39.499$ & 248 & 38.730 & $36.962,40.499$ & 252 \\
\hline
\end{tabular}

Numbers in cells are means, associated $95 \%$ confidence intervals, and useful sample size for each concept. Smoking support is the difference in encouragers by discouragers among observed social ties divided by the number of observed ties. Smoking models is the number of smokers among observed social ties divided by the number of observed ties

${ }^{a}$ Indicates a significant difference in means between men and women based on a $t$ test assuming unequal variances 
network smoked. Women and men encountered statistically equivalent $(t=0.19, p<0.85)$ amounts of smoking in their network, $47 \%$ (95\% CI 45-52) and 48\% (95\% CI 44-52), respectively.

About one-third (95\% CI 29-37) of respondents were current smokers. Smokers typically smoked 16.43 (95\% CI 14.78-18.07) cigarettes per day. Men reported a significantly $(t=15.60, p<0.01)$ higher, $61 \%$ (95\% CI 54-67), smoking prevalence than women, $6 \%$ (95\% CI 3-9), and male smokers smoked significantly $(t=2.87, p<0.01)$ more cigarettes, 17.28 (95\% CI 15.55-19.00), than women, 10.11 (95\% CI 5.22-15.00).

When smoking support increased by a factor of $10 \%$, women had a $73 \%$ (95\% CI 1.37-2.18) and men a $21 \%$ (95\% CI 1.07-1.37) higher odds of smoking, as shown in the first columns of Table 2. Women's smoking, consistent with expectations, had a stronger association with smoking support than men $(z=3.18, p<0.01)$. The presence of smoking models was unrelated to smoking in Seoul among women ([Odds ratio $(\mathrm{OR})]=1.11 ; 95 \%$ CI $0.89-1.40$ ) or men $(\mathrm{OR}=1.09 ; 95 \%$ CI $0.98-1.21)$. Any differences between the association of smoking models and smoking status between genders was not significant $(z=0.77, p<0.44)$.

The differences in the association between smoking support and smoking for men and women was clear; smoking among women increased more rapidly as a function of smoking support than it did among men, as shown in Fig. 1a. For example, an increase in smoking support from 75 to $25 \%$ net discouragement was associated with a $27 \%$ (95\% CI 10-49) higher probability of smoking among women compared to a $19 \%$ (95\% CI 8-27) higher probability among men. The difference in the probability of smoking among men and women was also evident at the intercept where men were much more likely to smoke.

Married women had a 95\% (95\% CI 0.01-0.43) lower odds of smoking than their unmarried counterparts, while married and unmarried men had similar odds of smoking. The interpretation, consistent with Cho et al. (2008), is that South Korean culture discourages married women from smoking but in ways not evident by our network indicators. For men younger than 30, each additional year of age was associated with a $50 \%$ (95\% CI 1.29-1.75) higher odds of smoking. Conversely, at or after 30 each additional year of age was associated with an $8 \%$ (95\% CI 0.89-0.95) lower odds of smoking. Age among women and education or occupation among both men and women were not associated with smoking in Seoul. Nonetheless, it appears that the combination of distal and proximate determinants and their interaction adequately predicted smoking status, as $73 \%$ of men's smoking status was correctly classified by the model [sensitivity $($ sen $)=82 \%$, specificity $($ spe $)=60 \%$ ] and $95 \%$ of women's smoking status was correctly classified (sen $=44 \%$, spe $=99 \%)$.

Smoking support was associated with a higher smoking rate $(\mathrm{B}=0.06 ; 95 \% \mathrm{CI} 0.01-0.10)$ among men as shown in the last column of Table 2. For the logged dependent variable, each unit change in the predictor variable may be interpreted as the percent difference in smoking rate (Wooldridge 2003). This suggests that for a $10 \%$ difference on the smoking support scale toward less discouragement, or more encouragement, men smoked 6\% (95\% CI 1-10) fewer cigarettes [i.e., $0.06=($ exponentiate $(0.06)-1)$ ]. Contrary

Table 2 Predictors of smoking among adults in Seoul, Korea, 2002

\begin{tabular}{|c|c|c|c|c|c|c|}
\hline \multirow{3}{*}{$\begin{array}{l}\text { Social support } \\
\end{array}$} & \multicolumn{4}{|c|}{ Smoking status } & \multirow{2}{*}{\multicolumn{2}{|c|}{$\begin{array}{l}\text { Smoking rate } \\
\text { Men }\end{array}$}} \\
\hline & \multicolumn{2}{|c|}{ Men } & \multicolumn{2}{|l|}{ Women } & & \\
\hline & $1.211^{* *}$ & $1.073,1.366$ & $1.726 * * *$ & $1.370,2.175$ & $0.057 *$ & $0.014,0.102$ \\
\hline Smoking models & 1.089 & $0.977,1.213$ & 1.112 & $0.885,1.397$ & 0.044 & $-0.008,0.097$ \\
\hline Years of education & 0.894 & $0.789,1.014$ & 1.051 & $0.747,1.478$ & -0.050 & $-0.111,0.009$ \\
\hline Married & 1.237 & $0.477,3.210$ & $0.068^{* *}$ & $0.011,0.427$ & 0.247 & $-0.149,0.643$ \\
\hline Professional & 0.381 & $0.125,1.159$ & 2.839 & $0.467,17.252$ & -0.144 & $-0.615,0.326$ \\
\hline Laborer & 0.765 & $0.188,3.107$ & 4.598 & $0.329,64.320$ & -0.255 & $-0.826,0.317$ \\
\hline Student & 1.065 & $0.279,4.067$ & 0.586 & $0.025,13.956$ & -0.137 & $-0.740,0.465$ \\
\hline Age & $1.502 * * *$ & $1.289,1.750$ & 1.277 & $0.935,1.742$ & 0.041 & $-0.027,0.110$ \\
\hline Age+ & $0.921 * * *$ & $0.889,0.954$ & 0.932 & $0.842,1.031$ & $-0.027 * * *$ & $-0.044,-0.012$ \\
\hline Constant & - & & - & & $2.552 *$ & $0.611,4.493$ \\
\hline Nagelkerke or OLS $R^{2}$ & 0.337 & & 0.514 & & 0.147 & \\
\hline$N$ & 247 & & 246 & & 156 & \\
\hline
\end{tabular}

Numbers in cells are adjusted odds ratios (smoking status) or regression coefficients (natural logarithm), 95\% confidence intervals, and two-tailed probabilities $* p<0.05, * * p<0.01, * * * p<0.001$. Listwise deletion was used for analysis. Analysis of smoking rates among women was not feasible given the small number of women who reported smoking $(N=21)$. Age indicates a 1-year increase in age before 30 and Age + a 1 -year increase in age at and after 30 


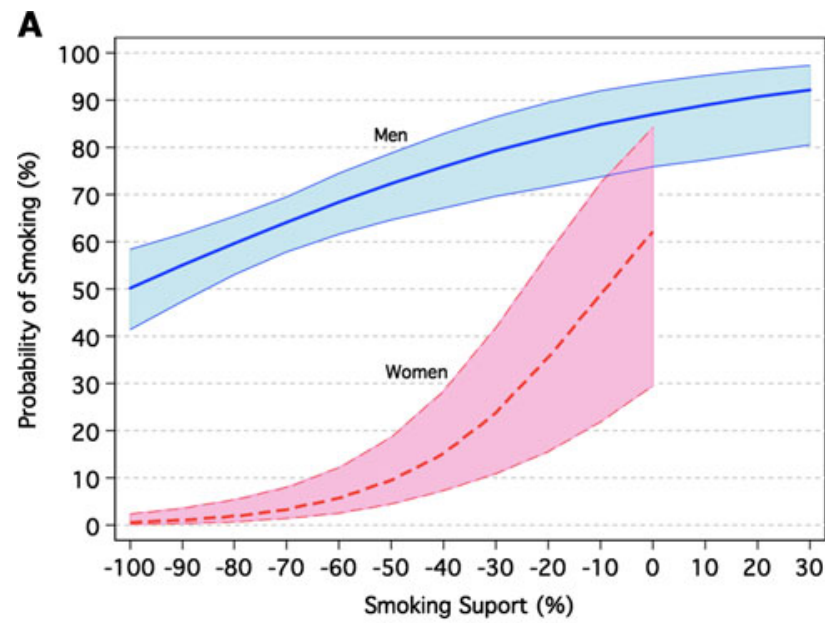

B

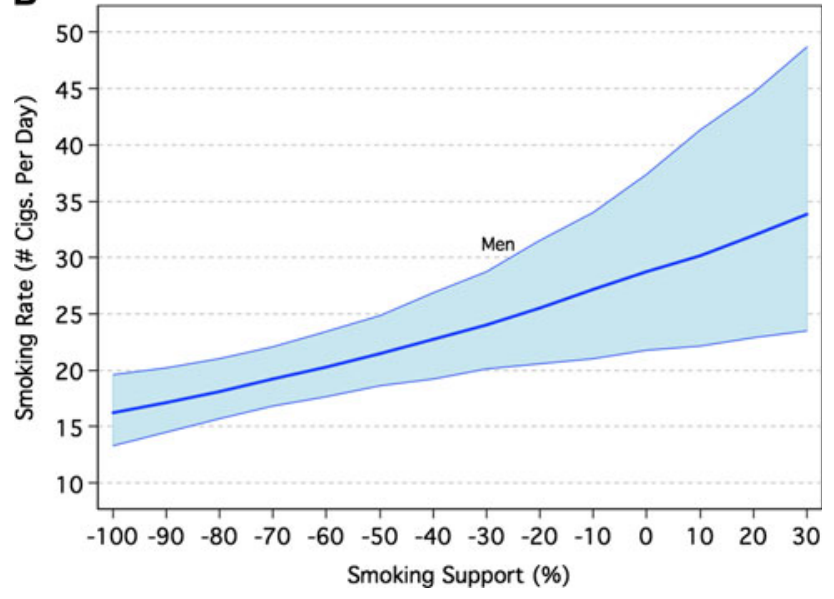

Fig. 1 Gender modifies the relationship between smoking support and smoking. a shows the predicted probability of smoking and b shows the expected smoking rate among current smokers with $95 \%$ confidence intervals by smoking support. Trends of increased smoking were statistically significant $(p<0.05)$ for all displayed associations

to expectations but consistent with earlier results in this study, the presence of smoking models was not significantly associated with men's smoking rate.

To clarify, an increase in smoking support from 75 to $25 \%$ net discouragement was associated with a $6.38(95 \%$ CI 0.86-12.30) higher smoking rate among men, more than a quarter of a pack per day, as shown in Fig. 1b. Over a year, a typical male South Korean would smoke about 2,329 (95\% CI 314-4,490) more cigarettes (about 116 packs) under a condition with $10 \%$ more encouragement, or less discouragement, in their network.

For men younger than 30, each additional year of age was not significantly associated with smoking rate. Each additional year in age at or after 30, however, was associated with a $3 \%(95 \%$ CI 1-4) lower smoking rate. About $15 \%$ of the variance in the $\log$ of smoking rate was explained by the model $\left(R^{2}=0.15\right)$.

\section{Discussion}

This analysis pursued an emerging avenue of investigation that seeks explanations for smoking from proximal and distal determinants, as well as their interaction. Consistent with expectations, smoking support was associated with smoking and smoking rate. However, beyond simple aggregate relationships, smoking support was more strongly associated with smoking among women, and women received more discouragement for smoking, than men.

The strengths of this study include a design and analysis strategy focused on social network mechanisms for smoking. Unlike studies that considered only models of a behavior, we discriminated between models and support, in the form of encouragement or discouragement of smoking, and found varying associations with smoking for each. Moreover, we incorporated theoretically meaningful moderated relationships by gender.

Weaknesses of this study include the relatively small cross-sectional survey $(N=500)$, restricting investigation of infrequent behaviors, self-reports subject to recall and reporting biases, and sampling from only the most metropolitan part of South Korea. The social network measures used a pre-generated list of family and friends and excluded other possible social influences of smoking behaviors. However, the list of alters centered around familial ties, which are the primary contacts in Asian cultures (Min 2001). Our measures of network mechanisms, while more advanced than studies that focus only on modeling, captured only episodic reinforcement for encouragement or discouragement, hence the underlying meaning or distribution of "discouragement" for respondents is unknown.

Prior studies of South Koreans' smoking provide important knowledge about the demography of smoking (Cho et al. 2004, 2006; Khang and Cho 2006; Khang et al. 2009), but the dynamics of smoking can be better understood by scrutinizing reinforcers in South Koreans' networks. Consider that many of these demographic variables, even those underscored as critical such as occupation, were not statistically significant after including social network mechanisms in the model.

The BEM and other ecological frameworks (Glass and McAtee 2006; Hovell et al. 2002, 2009; Link and Phelan, 1995; McLeroy et al. 1988) assert that levels of social context interact in complex ways. Unfortunately, at least in the peer influence literature, these interactions have been rarely reported (Smith and Christakis 2008; Berkman et al. 2000). The positive findings in this study suggest that focusing exclusively on the average association within samples misses critical and theoretically relevant moderation, and may result in serious inferential errors. Had we 
fitted a pooled model, for example, we would have overestimated the relationship between smoking support and smoking by $8 \%$ among men and underestimated the relationship by $24 \%$ among women.

Noteworthy studies of networks and smoking, like Christakis and Fowler (2008), report smoking is conditioned on the number of smoking models in their network. In this study, the discrimination between models and reinforcing generalities of encouragement or discouragement resulted in consistent null findings for smoking models. This non-finding may be a consequence of near ubiquitous smoking among the male South Korean population that results in smoking models having less influence. Or, reinforcers of smoking are not captured by the presence of a behavior; rather, how persons are motivated by explicit encouragement or discouragement for smoking may be what matters.

The gender differential in networks' association with smoking is relevant to studies of South Korean Americans. South Korean women do not have as high a level of independence as men, and smoking among women has been discouraged, as indicated by the large negative associations between female gender and smoking status in earlier studies (Cho et al. 2008). Yet, studies report a reversal in smoking rates by gender among South Korean Americans in which smoking rates dropped among men and increased among women (Hofstetter et al. 2004; 2007; CDC 2001; Lee 2000; Mermelstein 1999); interpreted as liberalizing and restraining shifts in smoking context for women and men. Our findings support these interpretations. In Seoul, women's networks were more anti-smoking and more strongly associated with smoking, compared to men; hence movement toward a mean tendency would result in less discouragement for women and thereby more smoking among women and the reversal for men.

In the South Korean context, especially in Seoul, genders may be becoming more equal. It is likely that cultural changes that move women to be more equal to men will have implications for how social influences impact women's smoking. Without changes in social norms that treat smoking as a sign of independence, women may be more likely to smoke as they gain equality with men. Khang and Cho (2006) found that from 1989 to 2003, the smoking prevalence increased among South Korean women who were 20-24 years old, the persons likely experiencing greater equality. This trend, combined with the findings reported here should alarm health advocates.

Using theories and analytic strategies focused on network mechanisms offers the advantage that these factors are modifiable. Interventions targeting social networks may reduce smoking among South Koreans even in the presence of many smoking models, as indicated by the consistent influence of smoking support and the impotence of smoking models. Specifically, programs that focus on social influence and the willingness to discourage persons who smoke may be most appropriate given the present findings. One study of South Korean Americans found that specific reports of social reprimand were strongly related to not smoking (Hofstetter et al. 2010). Such programs may wish to use health educators within South Korean communities to design workshops encouraging support for smoking cessation, directing interventions to the peers or social network members of South Korean smokers. Such a pattern is already in place for one of the most common addiction treatment regimes, alcoholics anonymous, where addicts are linked to a network were support for overcoming their addiction is promoted among clusters, not individuals.

This is a marked departure from the bio-medical model, where the person at risk or already smoking will be provided education or counseling to change in isolation of factors outside the individual. Such network based programs may wish to emphasize the appropriateness of reproaches since in South Korean culture women may feel uncomfortable asking male alters not to smoke. Social network interventions among South Koreans may be more effective than among other populations, since Asian cultures are somewhat more likely to choose behaviors that conform with the desires of others (Hofstede 1991; Markus and Kitayama 1991). While these programs could target smoking cessation, the findings concerning smoking rate suggest that even if cessation is not achieved, interventions may be successful in reducing the number of cigarettes smoked, though the beneficial effects of smoking reductions remain unclear (Tverdal and Bjartveit 2006).

It is also likely that government lead tobacco control will reduce smoking in South Korea (Goodman et al. 2009; Chung et al. 2009; Khang et al. 2009), and South Koreans appear to have strong public support for anti-smoking policies, at least in the work place (Halpern and Taylor 2009), but the implications of such changes on social networks is not well-studied. Though, we might expect such programs to have cascading effects where they promote fewer models of, and more discouragement of, smoking.

Future network studies of smoking may not find sufficient variance to explore mediating and moderating differences within a single society; instead, such hypothetical associations may require cross-cultural analysis (Ayers et al. 2010a, b). Alternatively, one might consider the pathways through which acculturation impacts other health predictors in immigrant populations (Ayers et al. 2009). Rather than assuming socio-demographic or cultural differences, studies should focus on the proximal determinants that result in these differences, and how proximal determinants behave 
differently according to socio-demographic or cultural differences. Ecological frameworks, like the BEM, offer potential for understanding and modifying risk practices through multi-level determinants, thereby reducing morbidity and mortality.

Acknowledgment This research was supported by funds provided by the Tobacco-Related Disease Research Program, Grant number 9RT-0073 to C. Richard Hofstetter and intramural support from affiliated departments and centers to John W. Ayers. We thank the editorial board, anonymous referees, David Jernigan, Hee-Soon Juon, Carl Latkin, Erin Thomas and Larry Wissow for helpful criticisms.

Open Access This article is distributed under the terms of the Creative Commons Attribution Noncommercial License which permits any noncommercial use, distribution, and reproduction in any medium, provided the original author(s) and source are credited.

\section{References}

Ayers JW, Hofstetter CR, Usita P, Irvin V, Kang S, Hovell MF (2009) Sorting out the competing effects of acculturation, immigrant stress, and social support on depression: a report on Korean women in california. J Nerv Ment Dis 197:742-747

Ayers JW, Hofstetter CR Hughes SC, Latkin C, Juon H-S, Irvin V, Park H, Paik HY, Hovell MF (2010a) Smoking on both sides of the Pacific: social networks and second hand smoke exposure among Korean nonsmokers in Seoul and California. Prev Med (under review)

Ayers JW, Hofstetter CR Hughes SC, Park H, Paik HY, Irvin V, Lee J, Juon H-S, Latkin C, Hovell MF (2010b) Smoking on both sides of the Pacific: home smoking restrictions and secondhand smoke exposure among Korean adults and children in Seoul and California. Nicotine Tob Res (under review)

Bandura A (1989) Social cognitive theory. In: Vasta R (ed) Annals of child development, vol 6. JAI Press, Greenwich, pp 1-60

Baruch Y (1999) Response rate in academic studies a comparative analysis. Hum Relat 52:421-438

Berkman LF, Glass T, Brissette I, Seeman TE (2000) From social integration to health: Durkheim in the new millennium. Soc Sci Med 51:843-857

Brambor T, Clark WR, Golder M (2006) Understanding interaction models: improving empirical analyses. Political Anal 14:63-82

Carroll RJ, Ruppert D (1988) Transformation and weighting in regression. Chapman and Hall, New York

Centers for Disease Control, Prevention (2001) Cigarette smoking among adults-United States, 1999. Morb Mortal Wkly Rep 50:869-873

Chang S (2004) The concept development of smoking temptation. J South Korean Acad Nurs 34:160-171

Cho HJ, Song YM, Davey Smith GD, Ebrahim S (2004) Trends in socio-economic differentials in cigarette smoking behavior between 1990 and 1998: a large prospective study in South Korean men. Public Health 118:553-558

Cho HJ, Khang YH, Yun SC (2006) Occupational differentials in cigarette smoking in South Korea: Findings form the 2003 social statistics survey. J Prev Med Pub Health 39:365-370

Cho H-J, Khang YH, Jun HJ, Kawachi I (2008) Marital status and smoking in Korea: the influence of gender and age. Soc Sci Med 66:609-619

Christakis NA, Fowler JH (2008) The collective dynamics of smoking in a large social network. N Engl J Med 358:2249-2258
Chung W, Kim H, Lim S, Lee S, Cho K (2009) Factors influencing cigarette smoking and quantified implications for anti-smoking policy: evidence from South Korea. Int J Public Healh 54:409419

Corrao MA, Guindon GE, Sharma N, Shokoohi DF (2000) Tobacco control country profiles. American Cancer Society, Atlanta

DeGrandpre RJ (2000) A science of meaning. Can behaviorism bring meaning to psychological science? Am Psychol 55:721-739

Do YK, Park K (2009) Local governments' dependence on tobacco tax revenue: a deterrent to tobacco control in the Republic of Korea. Bull World Health Organ 87:692-699

Frey JH (1989) Survey research by telephone. Sage Publications, Thousand Oaks

Gallup Korea (2001) Survey on the smoking prevalence. The Korea Gallup Report, Washington, DC

Glass TA, McAtee MJ (2006) Behavioral science at the crossroads in public health: extending horizons, envisioning the future. Soc Sci Med 62:1650-1671

Glenn SS, Ellis J, Greenspoon J (1992) On the revolutionary nature of the operant as a unit of behavioral selection. Am Psychol 47:1329-1329

Goodman PG, Haw S, Kabir Z, Clancy L (2009) Are there health benefits associated with comprehensive smoke-free laws. Int J Public Healh 54:367-378

Ha BM, Yoon SJ, Lee HY, Ahn HS, Kim CY, Shin YS (2003) Measuring the burden of premature death due to smoking in Korea from 1990 to 1999. Public Health 117:358-365

Halpern MT, Taylor H (2009) Beliefs regarding smoking in the workplace: results from the Global Workplace Smoking Survey. Int J Public Healh 54:391-401

Hofstede G (1991) Cultures and organizations: software of the mind. McGraw-Hill, London

Hofstetter CR, Hovell MF, Lee J, Zakarian J, Park H, Paik HY, Irvin V (2004) Tobacco use and acculturation among Californians of South Korean descent: A behavioral epidemiological analysis. Nicotine Tob Res 6:481-489

Hofstetter CR, Hovell MF, Jung KR, Raman R, Irvin V, Ni R (2007) The first puff: forces in smoking initiation among Californians of South Korean descent. Nicotine Tob Res 9:1277-1286

Hofstetter CR, Hovell MF, Irvin V, Ayers JW, Hughes SC, Kang S (2010) It's others, not the police: smoking, reprimand, and fines among adults of South Korean descent in California. Health Psych (in press)

Hovell MF, Hughes SC (2009) The behavioral ecology of secondhand smoke exposure: a pathway to complete tobacco control. Nicotine Tob Res 11:1254-1264

Hovell MF, Wahlgren DR, Gehrman CA (2002) the behavioral ecological model: integrating public health and behavioral science. In: DiClemente RJ, Crosby RA, Kegler M (eds) Emerging theories in health promotion practice and research: Strategies for improving public health. Jossey-Bass Inc., San Francisco, pp 347-385

Hovell MF, Wahlgren DR, Adams MA (2009) The logical and empirical basis for the behavioral ecological model. In: Diclemente RJ, Crosby RA, Kegler M (eds) Emerging theories in health promotion practice and research: Strategies for improving public health, 2nd edn. Jossey-Bass, San Francisco, pp 415-449

Jee SH, Ohrr H, Kim IS (1999) Effects of husbands' smoking on the incidence of lung cancer in South Korean women. Int J Epidemiol 28:824-828

Jee SH, Samet JM, Ohrr H, Kim JH, Kim IIS (2004) Smoking and cancer risk in Korean men and women. Cancer Causes Control 15:341-348

Ji M, Hofstetter CR, Hovell MF, Irvin V, Song YJ, Lee J, Park H, Paik HY (2005) Smoking cessation patterns and predictors 
among adult Californians of South Korean descent. Nicotine Tob Res 7:59-69

Kang HY, Kim HJ, Park TK, Jee SH, Nam CM, Park HW (2003) Economic burden of smoking in Korea. Tob Control 12:37-44

Khang YH, Cho HJ (2006) Socioeconomic inequality in cigarette smoking: trends by gender, age, and socioeconomic position in South Korea, 1989-2003. Prev Med 42:415-422

Khang YH, Yun SC, Cho HJ, Jung-Choi K (2009) The impact of governmental antismoking policy on socioeconomic disparities in cigarette smoking in South Korea. Nicotine Tob Res 11:262269

King G, Tomz M, Wittenberg J (2000) Making the most of statistical analyses: improving interpretation and presentation. Am J Pol Sci 44:341-355

Korean Association of Smoking and Health (2001) Reasons why the rate of smoking is increasing in Korea. Available via http://www.nosmokeguide.or.kr/board/earth_view.asp?board_code= B1\&idxno=2\&find_chk=\&find_str=8. Accessed 14 Dec 2004

Kraemer HC, Frank E, Kupfer DJ (2006) Moderators of treatment outcomes clinical, research, and policy importance. J Am Med Assoc 296:1286-1289

Lee SY (2000) A Study on the Factors Influencing Smoking Behavior in Korea. J South Korean Soc Health Stat 25:41-50

Lee SW (2003) The effects of the smoking \& drinking on tv on health related behavior. Kwan Dong University Press, Seoul

Link BG, Phelan J (1995) Social conditions as fundamental causes of disease. J Health Soc Behav extra issue, pp 80-94

Markus HR, Kitayama S (1991) Culture and the self: implications for cognition, emotion, and motivation. Psych Rev 98:224-253

Marsden PV (1990) Network data and measurement. Annu Rev Sociol 16:435-463

Marsden PV (2005) Recent developments in network measurement. In: Scott PJ, Wasserman S (eds) Models and methods in social network analysis. Cambridge University Press, New York, pp 8-30
Marsh L, Cormier DR (2001) Spline regression models. Sage Publications, Thousand Oaks

McLeroy KR, Bibeau D, Steckler A, Glanz K (1988) An ecological perspective on health promotion programs. Health Educ Behav 15:351-377

Mermelstein R (1999) Explanations of ethnic and gender differences in youth smoking: a multi-site, qualitative investigation. Nicotine Tob Res 1:S91-S98

Min PG (2001) Changes in South Korean immigrants' gender role and social status, and their marital conflicts. Sociol Forum 16:301-320

Poland B, Frohlich K, Haines RJ, Mykhalovskiy E, Rock M, Sparks R (2006) The social context of smoking: the next frontier in tobacco control? Tob Control 15:59-63

Reifman A, Watson WK, McCourt A (2006) Social networks and college drinking: probing processes of social influence and selection. Pers Soc Psychol Bull 32:820-832

Schroeder S (2008) Stranded in the periphery-the increasing marginalization of smokers. N Engl J Med 358:2284-2286

Smith KP, Christakis NA (2008) Health and social networks. Annu Rev Sociol 34:405-429

STAT-KOREA (2002) Census. Available via http://www.stat.go. kr/statcms/main.jsp. Accessed 6 March 2006

Tverdal A, Bjartveit K (2006) Health consequences of reduced daily cigarette consumption. Tob Control 5:472-480

U.S. Department of Health and Human Services (1996) National Center for Health Statistics. NHANES III reference manuals and reports (CD-ROM). Centers for Disease Control and Prevention, Hyattsville, MD

Watson JB (1994) Psychology as the behaviorist views it. Psycho Rev 101:248-253

Wooldridge JM (2003) Introductory econometrics: a modern approach, 2nd edn. Thomson South-Western, Mason

World Health Organization (2002) Factsheet: smoking statistic. Available via http://www.wpro.who.int/media_centre/fact_sheets/ fs_20020528.htm. Accessed 2 Feb 2008 